\title{
Simulation-Assisted Building Energy Performance Improvement Using Sensible Control Decisions
}

\author{
M.F. Pichler, A. Dröscher, H. Schranzhofer \\ Graz University of Technology, Institute of Thermal Engineering. \\ martin.pichler@tugraz.at, angela.droescher@tugraz.at, hermann.schranzhofer@tugraz.at \\ G.D. Kontes, G.I. Giannakis, E.B. Kosmatopoulos, and D.V. Rovas \\ Department of Production Engineering and Management, Technical University of Crete, Chania, Greece, and \\ Informatics \& Telematics Institute, Center for Research and Technology-Hellas (ITI-CERTH), Thessaloniki, Greece. \\ gkontes@iti.gr, ggiannakis@isc.tuc.gr, kosmatop@dssl.tuc.gr, rovas@dpem.tuc.gr
}

\begin{abstract}
The building sector contributes significantly to global energy consumption and emission of greenhouse gases. Thermal insulation along with installation of energy-efficient building systems can reduce energy needs while preserving or improving occupant comfort levels. Still sensible control decisions, to harmoniously and effectively operate all building thermal systems, can be used to further improve building energy performance and/or thermal comfort. In this article, a simulation-assisted methodology is presented to automatically generate such decisions. There are two ingredients to our approach: a thermal simulation model - a surrogate of the real building - used to evaluate the effects of potential decisions; and, a cognitive adaptive optimization algorithm used to intelligently search for the "best" control decision. A user-defined cost function is used to compare various decision strategies. Corroborating simulation results are presented to quantify the expected benefits of the proposed approach.
\end{abstract}

\section{Categories and Subject Descriptors}

J.2 [Physical Sciences and Engineering]: Engineering; I.2.8 [Problem Solving, Control Methods, and Search]: Control theory; J.7 [Computers In Other Systems]: Process control

\section{General Terms}

Algorithms, Performance, Management

\section{Keywords}

Energy Efficiency in Buildings, Adaptive Optimization,

Permission to make digital or hard copies of all or part of this work for personal or classroom use is granted without fee provided that copies are not made or distributed for profit or commercial advantage and that copies bear this notice and the full citation on the first page. To copy otherwise, to republish, to post on servers or to redistribute to lists, requires prior specific permission and/or a fee.

BuildSys'11, November 1, 2011, Seattle, WA, USA

Copyright 2011 ACM 978-1-4503-0749-9 ...\$10.00
Large-Scale Systems, Non-Linear Systems

\section{Introduction}

The European target of reduction of greenhouse gases by $20 \%$ until 2020 while allowing economic and demographic growth, is unattainable unless certain measures are taken. In 2008, the Total Final energy Consumption (TFC) of the EU 27 amounted to $49 \mathrm{EJ}$, with the Household sector contributing to $25.4 \%$ of the TFC on average and with the inclusion of the Services sector bringing the contribution up to $40.8 \%$ of the TFC. Similar conclusions can be drawn for other regions of the world, suggesting the necessity to develop technologies and methods to reduce energy consumption in these sectors. According to [4], energy used for heating accounts to one-fifth and one-third of the TFC in the Household and Services sectors, respectively. Apart from public policy actions aimed at raising consumer awareness - i.e. turning building users into "prosumers" - other possibilities at mollifying the problem exist: first, optimization of the building envelope, and; second, improving effectiveness in utilization of energy. In the former case, many approaches exist and have been studied extensively in the literature; while in the latter, Building Energy Management Systems (BEMS) can be used to monitor and control the use of energy in the building. Studies show that smart and predictive control strategies are viable methods to reduce building thermal energy demand [13, 15].

In current practice, the majority of BEMS, use simplistic rule-based controllers. Alternative approaches such as predictive, intelligent comfort, and predictive weather-data based controllers have been suggested to tackle but also exploit the slow dynamics of thermal systems in buildings [2, 9, 12, 13] — with these approaches being in most cases data-driven. Progress in building technologies, weather forecasting and low-cost embedded computing systems pave the way for implementation of intelligent controllers in building control applications. The use of Model Predictive Control (MPC) has been suggested, e.g. in [6], as a viable option. In [13] practical experiments with passive cooling via MPC have been reported to reduce overheating by approximately 
$1 \mathrm{~K}$. Energy reduction while maintaining high user comfort by means of MPC has been investigated in the OptiControl Project, in which a theoretical potential of up to $40 \%$ reduction of Non-Renewable Primary Energy (NRPE) use is estimated [14]. In the presence of uncertainties, the expected benefits are smaller but, to date, there is little experimental evidence as to the actual savings potential.

\subsection{The PEBBLE Project}

In Project PEBBLE (Positive Energy Buildings through Better controL dEcisions), the goal is the development of model-based controllers, for the holistic operation of all energy-influencing systems in the building, and the deployment and evaluation of such strategies in three real-world large-scale office buildings. Simulation models for each of these buildings were created in TRNSYS, EnergyPlus and Modelica. The Cognitive Adaptive Optimization (CAO) algorithm is used to efficiently explore the large decision space and identify "good" controllers to be applied to the real building, with the goal of maximization of the "Net Expected Benefit" (NEB) [11]. For simple buildings, the potential of adapting the $\mathrm{CAO}$ algorithm for building control has been investigated in [8].

In this paper (parts of) a validated TRNSYS building model of a real building, the Center for Sustainable Building (ZUB) in Kassel, Germany is used and connected with the CAO algorithm. The cost function used to evaluate potential decisions depends on the simulated energy demand and the level of comfort which is measured using the Fanger index [1]. In Section 1.2 the ZUB building is described; the definition of the control problem along with the connection between the CAO algorithm and the TRNSYS model is described in Section 2, results obtained are presented in Section 3 , and, conclusions along with suggestions for future work in Section 4.

\subsection{Center for Sustainable Building (ZUB)}

The ZUB building, built in 2001, located at the campus of the University of Kassel, Germany is an exemplary lowenergy building. The ZUB has three floors, a basement and an atrium in contact with a nearby building. It is occupied by researchers in the field of building physics, equipped with a significant number of sensors, and used for experimental investigations in the field of energy optimization and building technologies.

The overall volume of the heavy-weight construction is $6882 \mathrm{~m}^{3}$, the net heated floor area is $1332 \mathrm{~m}^{2}$ and the main floor space is $892 \mathrm{~m}^{2}$ [16]. Floor height is close to $4 \mathrm{~m}$ and the annual heat demand is between that of a low-energy building and a passive house, approximately $30 \mathrm{kWh} / \mathrm{m}^{2}$ or $5.3 \mathrm{kWh} / \mathrm{m}^{3}$; electricity consumption based on the heated net floor area is approximately $20 \mathrm{kWh} /\left(\mathrm{m}^{2} \mathrm{a}\right)$ [7, 16]. The building has three Thermally Activated Building Systems (TABS): (i) a basement slab or ground heat exchanger; (ii) radiant floors, and; (iii) radiant ceiling systems.

For heating and cooling purposes, floor and ceiling TABS utilize district hot-water in the winter and cooling from the basement slab during summer. A central mechanical ventilation system with heat recovery and natural night-time ventilation with open windows in the atrium ensure in an energy- efficient manner the conditioning of offices and rooms. A window/façade ratio close to one, favorable building orientation, and adjustable shading devices provide for high solar gains during the winter months and prevent from solar overheating in the summer while maintaining a high-degree of solar utilization. There are approximately 1300 measurement points (temperature, humidity, energy flows etc.) throughout the building, making the validation of the thermal model described in the next Section possible. More details about the ZUB and its energy concept are given in [7, 16].

\section{Methodology}

To evaluate the effect of control actions on the building energy performance a thermal simulation model was created using the TRNSYS building simulation tool. A second ingredient is the CAO algorithm implemented in MATLAB. The dynamic interaction between the two components was established using a master-slave relationship with the MATLAB script invoking the TRNSYS simulation as needed. Selecting the "best" control strategy for the following day requires a few hundred calls to the simulation engine, so computational cost along with reliability and stability issues should be considered for real-world implementation. In the following the proposed approach is described in more detail.

\subsection{Building thermal simulation model}

Figure 1 shows the ZUB building model, designed using Google SketchUp, with shading groups in purple. Since TABS can be operated independently for each room, each physical room was defined as a separate thermal zone, for a total of 26 zones to model the whole building.

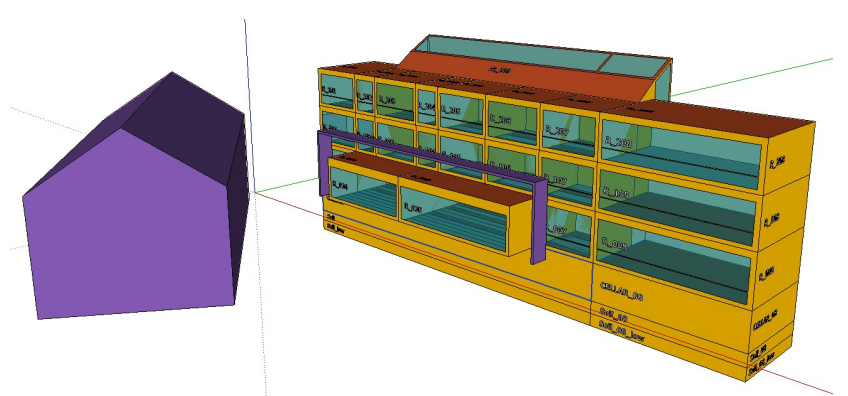

Figure 1. Whole ZUB building model designed using Google SketchUp, comprising 26 thermal zones.

To properly ascertain the effects of primary beam, diffuse, and long-wave radiation within each zone, detailed geometric characteristics are needed. The detailed radiation model selected uses semi-analytic methods for the view factor calculations. 1

The atrium at the back of the building consists of three thermal (convective) air-nodes, - one per floor, - combined to a single (radiative) zone. This new feature simplifies the simulation of thermal stratification.

A detailed simulation for each zone, while necessary for validation purposes, is too detailed for the needs of designing the decision strategies. For this reason a reduced-model

\footnotetext{
${ }^{1}$ The model used is available since TRNSYS version 17, and has the limitation that each thermal zone should be closed and convex.
} 
was created, shown in Figure 2, and is essentially a cutout of the building that preserves all relevant characteristics from the whole model - this model will be called the Tower in the following. Below the basement, two additional thermal zones constitute the soil bed that thermally interacts with the building, especially during base slab operation. Thus, the model has seven real and two auxiliary thermal zones, and the atrium zone comprises three thermal air-nodes:

- soil bed below basement (SOIL) and (SOIL_LOW),

- basement (CELLAR),

- ground, first and second floor (R007), (R107), (R207),

- atrium (AT009, AT109, AT209).

Figure 2 shows the Tower with the same shading groups as for the whole building. In addition the jutty on the ground floor, is not included in the thermal model of the Tower, and is modeled as a shading group. The blue colored walls of the

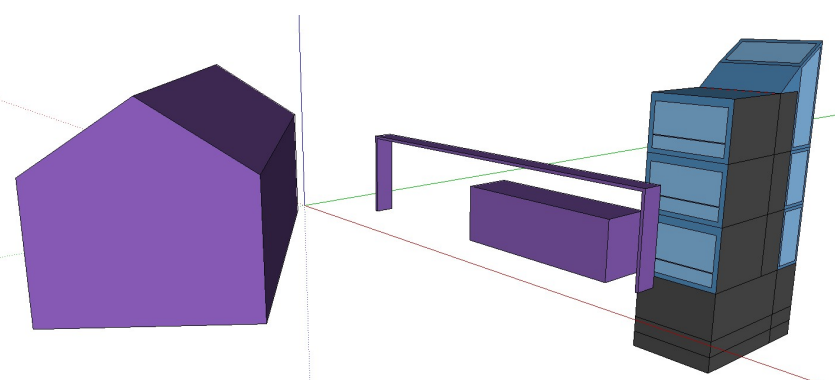

Figure 2. Tower and shading groups used in the thermal simulation model.

Tower indicate external walls; the dark-gray walls are defined as adiabatic walls permitting no heat flux across the walls. This is only an approximation, however, available measurement data showed only small temperature deviations in neighboring rooms ( $\pm 5 \mathrm{~K}$, depending on the season), and using these measurement data as boundary conditions did not lead to significantly different results. More details on the validation of the model can be found in [3].

As mentioned earlier, the building is equipped with three different TABS. The mass flow of the heating/cooling fluid and the flow temperature (in case of heating) are model input parameters.

Airflows in the building are modeled using TRNFlow, which is the integration of COMIS into TRNSYS. COMIS is a multi-zone air flow and contaminate transport model developed within IEA-Annex 20 and evaluated in Annex 23 [5]. The TRNFlow network consists of thermal (indoor) and external (outdoor) air-nodes connected via air-links allowing for simulation of natural ventilation. The air-links are used to model large openings (windows, doors, etc.) and cracks (ventilation bypass for the office-doors). Air-links are characterized by their dimensions, discharge coefficients, and opening factors, the latter are model parameters existing only for large openings. The mechanical ventilation system is modeled with fans, ducts and other equipment stringed together via auxiliary air-nodes, which can be subject to defined conditions in terms of temperature and humidity.

The building model, flow parameters of TABS, opening factors of windows and doors, and behavior under certain conditions were validated using available measured data.

\subsection{Simulation set-up}

The set-up depicted in Figure 3 comprises two major parts: the MATLAB environment (instance 1), and the TRNSYS simulation (simulation.dck), represented by the upper left and lower box, respectively. MATLAB (instance 1) includes the CAO algorithm, a parameter file and the simulation call. The TRNSYS simulation embraces weather data, TRNSYS Type155, and the whole building model including basic parameters such as geometric and wall definitions. Type155 represents an embedded MATLAB environment, indicated as (instance 2). Data exchange between the two MATLAB instances is realized by means of *.mat files.

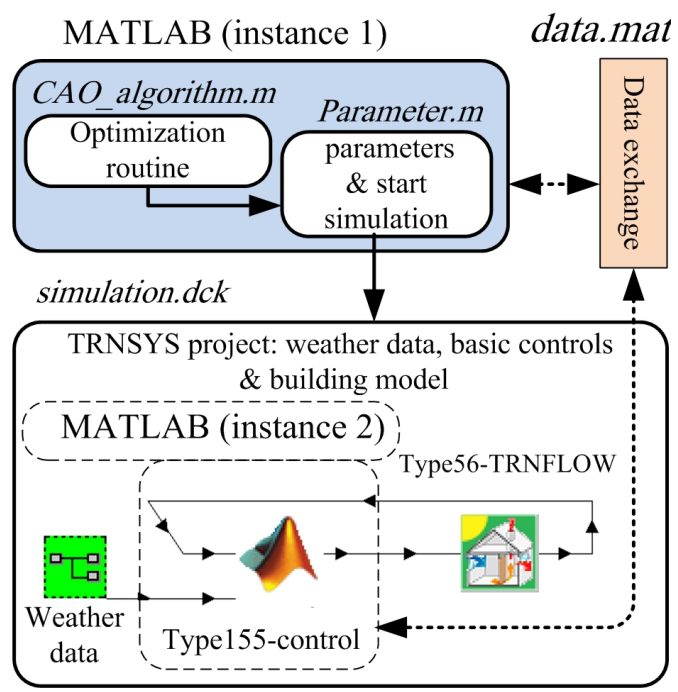

Figure 3. Simulation set-up: Illustration how MATLAB (instance 1) calls TRNSYS and interacts with MATLAB (instance 2) being part of the TRNSYS simulation.

The building parameters and physical constants that are required by Type 56 are either given as constant values or as input-variables to be supplied by Type155. In addition to the specification of certain building parameters, Type155 supplies schedules or provides control actions to be expected in reality. Supplied schedules include occupancy profiles and internal gains, window or mechanical ventilation operation, external shading control, to mention but a few. Type155 is essentially a MATLAB script; it allows the inclusion of individual device control rules and furthermore opens an interface for more complex controls such as the ones used here. Although the occupancy profile is input as a schedule, for future real operation this profile could be supplied dynamically from a database, using values recorded from in-building sensors.

Establishing the two-way interaction whereby dynamic schedules created from the CAO algorithm are passed to TRNSYS is a prerequisite for the development of the control strategies. A second requirement is the polling of weather forecasts so that weather files can be created for the simulation. The procedure of parameter search for certain con- 
trollers being part of the building, is named an experiment. An experiment is conducted for a certain prediction horizon. In a typical control design scenario the CAO algorithm calls the TRNSYS simulation a few hundred times during one experiment, each time to simulate for the prediction horizon of one day - of course, the number of times TRNSYS needs to be invoked hinges upon the complexity of the building and the starting initial guess for the controller.

\subsection{Cognitive Adaptive Optimization}

To assess the performance of the CAO algorithm assume a controller is to be designed to control ideal cooling in three thermal zones of the building. A simple proportional controller of the form:

$$
U=\theta X,
$$

is assumed; here $U=\left[u_{1}, u_{2}, u_{3}\right]^{T}$ are the control actions shown in Table 1] $X=\left[x_{1}, \ldots, x_{9}\right]^{T}$ are the building states shown in Table 2 and $\theta \in \mathbb{R}^{3 \times 9}$ is a matrix, to be determined, containing the controller gains.

Table 1. Control actions

\begin{tabular}{|c|c|c|}
\hline Control Action & Description & Range \\
\hline$u_{1}$ & T setpoint of Zone R007 & {$[20-30]^{\circ} \mathrm{C}$} \\
\hline$u_{2}$ & T setpoint of Zone R107 & {$[20-30]^{\circ} \mathrm{C}$} \\
\hline$u_{3}$ & T setpoint of Zone R207 & {$[20-30]^{\circ} \mathrm{C}$} \\
\hline
\end{tabular}

Table 2. Controller state variables

\begin{tabular}{|c|c|}
\hline Variable & Description \\
\hline$x_{1}$ & Outside Temperature \\
\hline$x_{2}$ & Outside Relative Humidity \\
\hline$x_{3}$ & Radiation on Window Façade \\
\hline$x_{4}$ & Tair of Zone R007 \\
\hline$x_{5}$ & Relative Humidity of Zone R007 \\
\hline$x_{6}$ & Tair of Zone R107 \\
\hline$x_{7}$ & Relative Humidity of Zone R107 \\
\hline$x_{8}$ & Tair of Zone R207 \\
\hline$x_{9}$ & Relative Humidity of Zone R207 \\
\hline
\end{tabular}

As part of the optimization process a series of candidate controllers $\theta_{c} \in \Theta_{c}$ is created. To evaluate and compare performance the following cost function is used:

$$
J=w \tanh \left(A_{1} E^{2}+B\right)+(1-w) \tanh \left(A_{2} \sum_{i=1}^{3} \frac{F_{i}^{2}}{3}+B\right) ;
$$

here $E$ is the total energy demand per time-step (i.e. power); and $F_{i}$ is the Fanger PPD index [1] in zone $i \in\{1, \ldots, 3\}$. The $F_{i}$ take values in the range $[5 \%-100 \%]$ representing the percentage of persons dissatisfied due to poor thermal comfort conditions prevailing in the zone.

A trade-off between energy and thermal comfort exists: on one hand, good thermal comfort requires maintaining zone conditions within strict limits, and this typically requires increased use of the HVAC systems and more energy; on the other hand, permitting slight discomfort (to within ranges set by comfort standards) can mean that less conditioning is required and therefore more energy can be saved. In the proposed cost function in Equation $2 w$ is a userdefined weight to adjust the relative importance between energy $(E)$ and thermal comfort $\left(F_{i}\right)$; here $w=0.1$ was used. In $2 B=2$ was selected so that Fanger index values over $30 \%-40 \%$ (high discomfort) are strongly penalized while values between $5 \%-15 \%$ (reasonable discomfort) are tolerated; see Figure 4. Finally, $A_{1}, A_{2}$ are normalization constants for $E$ and $F$, respectively.

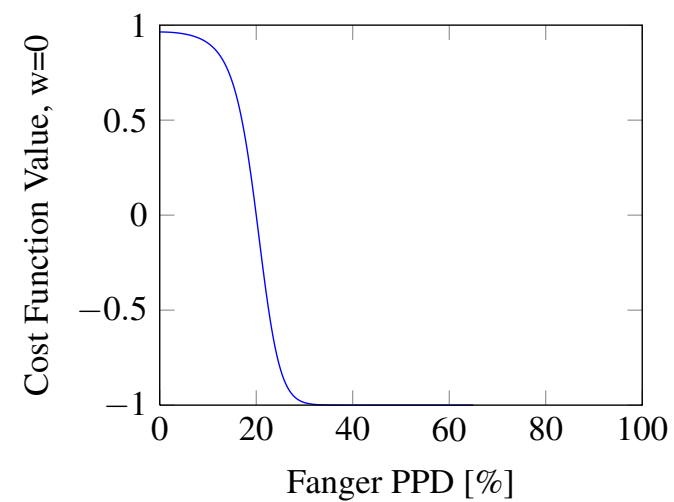

Figure 4. Sigmoid cost function

To design a proper controller for the building, the CAO optimization process starts and, using weather and occupancy forecasts for the prediction horizon, a controller (i.e. a set of proper gains $\theta$ ) is designed. The CAO [8] algorithm is a local search algorithm, used to improve an initial controller provided by an expert or by an Approximate Optimal Control design process (AOC) such as in [10].

\begin{tabular}{|c|c|}
\hline \multicolumn{2}{|c|}{ 1: Input: $\theta_{0}, K, N, K_{1} / /$ Initial Controller } \\
\hline 2: & Repeat for $k=0: K_{1}-1 / /$ Initial exploration \\
\hline 3: & $J_{k}=J\left(\theta_{k}\right) / /$ Run using the simulator \\
\hline 4: & end \\
\hline 5: & Repeat for $k=K_{1}: K-1$ \\
\hline 6: & $J_{k}=J\left(\theta_{k}\right) / /$ Run on the simulator \\
\hline 7: & $\hat{J}_{k}\left(\theta_{k}\right)=\vartheta_{k}^{\tau} \phi\left(\theta_{k}\right) / /$ Construct the estimator \\
\hline 8: & $\bar{\theta}=\underset{\theta_{k}}{\operatorname{argmin}} J_{k} / /$ The best controller so far \\
\hline 9: & $\begin{aligned} \Theta_{c}=\left\{\theta_{c}^{(j)} \mid \bar{\theta}+\alpha \zeta_{k}^{(j)}, j \in\{0, \ldots, N\}, \ldots\right. \\
\left.\ldots \zeta_{k}^{(j)} \sim \mathcal{N}(0,1)\right\}\end{aligned}$ \\
\hline 10: & $\theta_{k+1}=\underset{\theta_{c} \in \Theta_{c}}{\operatorname{argmin}} \hat{J}_{k}\left(\theta_{c}\right) / /$ Select the best \\
\hline 11: & end \\
\hline 12: & $J_{K}=J\left(\theta_{K}\right)$ \\
\hline & put: $\theta=\underset{\operatorname{argmin}}{\arg } J_{K}$ \\
\hline
\end{tabular}

Table 3. Cognitive adaptive optimization

A sketch of the algorithm is shown in Table 3 Line 1 provides an initial controller to the algorithmic framework 
for further improvement. In Lines $2-4, K_{1}$ controllers are created, exploring the area around the initial controller, and are evaluated using the cost function in (2) via simulations in TRNSYS (simulator). In Line 7 a Support Vector Machine (SVM) estimator [17] is created using the controllers evaluated on the simulator so far and their corresponding cost function values, and in Line 8 the "best" controller $\bar{\theta}$ (according to the cost function) among them is selected. With this controller, in Line 9 a set of approximately 1000 candidate controllers is created. In Line 10 each candidate controller $\theta_{c} \in \Theta_{c}$ is evaluated using the estimator ${ }^{2}$ and the best controller is selected for evaluation on the simulator in the next iteration of the algorithm. When $K$ iterations are executed, in Line 12 the best controller of the experiment is determined. In practice, this would be the controller for the real building the following day and state variables would be measurement data obtained from the in-building sensors.

\section{Results and discussion}

To test the CAO and TRNSYS connection, and to assess the performance of the $\mathrm{CAO}$ algorithm, the following building control design task is considered: variation of the building-zone setpoints of an ideal AC system, to minimize energy while maintaining acceptable comfort levels. Occupancy in Zones R107 and R207 is assumed to be from 9:0017:30, allowing for a $45 \mathrm{~min}$ lunch break at 12:30, with 3 people in each zone; zone R007 is assumed to be unoccupied for the day of the experiment. Occupancy and weather predictions are assumed to be exact. Real weather data of a hot summer day in Kassel (August 10 ${ }^{\text {th }}, 2001$ ) were used, see Figure 5 .

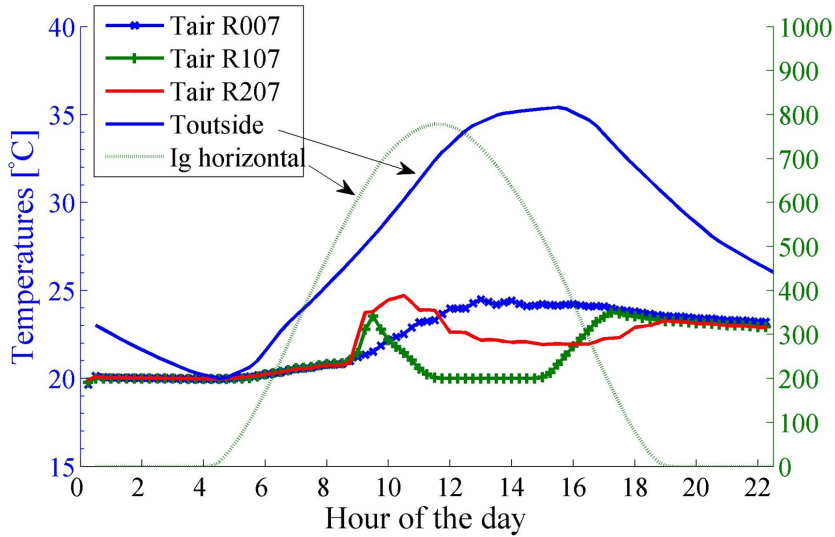

Figure 5. Inside and outside temperatures (left axis) and global horizontal radiation (right axis).

Upon application of the CAO algorithm to find the best $\theta$, the building response obtained is shown in Figures 5 , 6 and 7 As people enter the building and the Fanger index increases (09:00-10:00, Figure 6), the controller cools zones R107 and R207 (Figure 7), while in zone R007 the temperature is free floating as there is no occupancy. When people exit the building for the night, the controller shuts-down the AC systems (since it does not have information about the

\footnotetext{
${ }^{2}$ Invoking TRNSYS for the evaluation would incur unmanageable computational costs.
}

next day and is unable to define strategies like night cooling), to save energy (Figure 7). Moreover, the possibility to save energy during lunch break is identified as there are no occupants inside the building. The controller changes (increases) the setpoints but does not completely turn off the cooling, since people re-enter the building after lunch break. Note here, that the algorithm designs the controller using only occupancy prediction and without any information regarding actual building schedules.

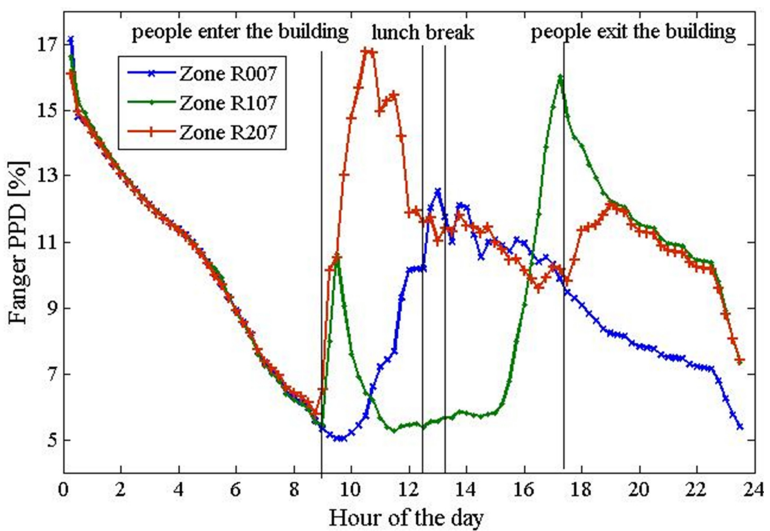

Figure 6. PPD characteristics for the best controller found $\left(\theta_{K}\right)$.

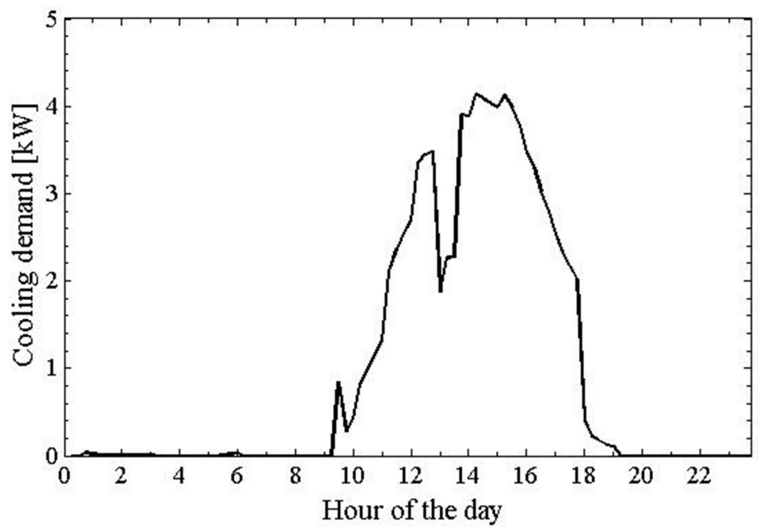

Figure 7. Useful cooling energy demand during one day with CAO operation.

To evaluate the potential energy savings of the controller, two rule-based controllers are defined: one that cools the rooms at constant temperature during the 24-hour period (blue line in Figure 8) and one that cools the rooms during the day only (red line in Figure 8). These controllers are compared to four controllers produced by the $\mathrm{CAO}$ algorithm (circles in Figure 8) with respect to total energy demand, and average Fanger PPD values while the zone is occupied. The different controllers generated by the optimization process indicate convergence to different local optima. A Pareto front is formed by the two static rule-based controllers (red and blue lines in Figure 8), defining a limit in their performance. 
In all cases the $\mathrm{CAO}$ algorithm performs better, leading to a theoretical energy saving potential for this simple example varying from $5 \%-14 \%$, while maintaining comfort at similar levels. This is due to the fact that the controller produced is allowed to vary the setpoints for the ACs during the day - rather than using a constant value.

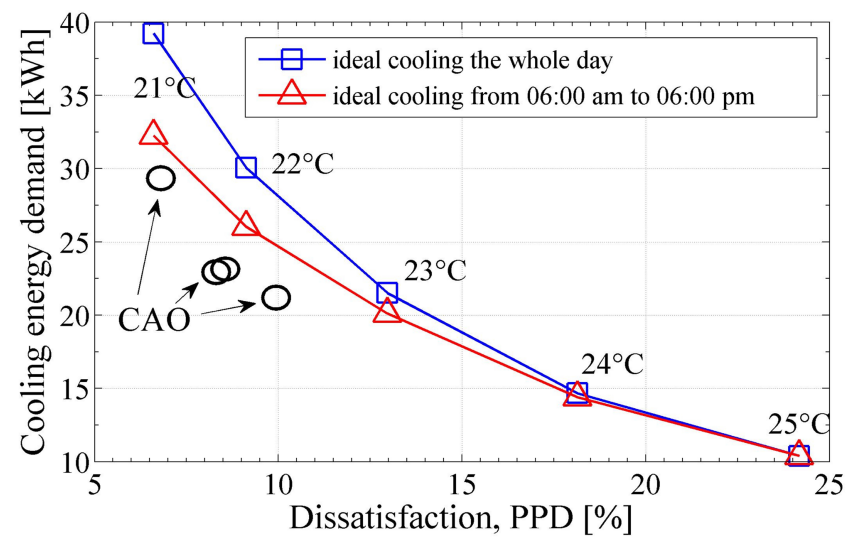

Figure 8. Control performance chart demonstrating the advantage of CAO algorithm against static controllers.

Type 155 in Figure 3 was operated non iteratively, which significantly decreased the primary computing time. Approximately $200-500$ iterations are required to reach a local optimum, starting with a naïve controller $(\theta=\mathbf{0})$. Not every experiment leads to the same local optimum, but in all cases a performance improvement is obtained. Each iteration lasts approximately $25 \mathrm{sec}$ (on an Intel Core 2 Duo CPU, 2.66 $\mathrm{GHz} \mathrm{CPU}$ ), thus an experiment takes between $1.4-3.5 \mathrm{~h}$.

\section{Conclusions and Future Work}

In the present work a stochastic optimization algorithm is linked to TRNSYS, so that energy performance in building operation can be optimized. The controller produced by the optimization process exhibits intelligent behavior, by saving energy during the night and during occupant's lunch break, and outperforms two static rule-based controllers acting upon the same building, leading to 5\%-14\% energy savings, while keeping comfort at acceptable levels.

The present work will be extended to the full building (simulated and real) with enhanced control features, such as day-night mechanical and natural ventilation, cool ceiling, etc. as has been investigated in [13]. This increased complexity require careful selection of the starting point for the optimization and the computational complexity will increase. The performance of the CAO algorithm will be compared with results obtained from a standard optimization tool (e.g. GENOPT) in a future paper. Furthermore, the parameters of the cost function need to be investigated systematically, to ensure scalability to buildings of higher complexity. Additionally in future work we address how to realize occupancy predictions.

\section{Acknowledgments}

This research was supported in part by the PEBBLE Project, funded by the European Commission FP7-ICT2007-9.6.3, ICT for Energy Efficiency, under contract \#248537. Special thanks go also to the colleagues Michael Krause and Juan Rodriguez Santiago from the FIBP in Kassel, for providing us with measurement data and other supporting activities.

\section{References}

[1] ASHRAE. ASHRAE Standard, ANSI/ASHRAE Standard 55-2004: thermal environmental conditions for human occupancy. 2004.

[2] J. Bai, S. Wang, and Z. Xiaosong. Development of an adaptive smith predictor-based self-tuning pi controller for an hvac system in a test room. Energy and Buildings, 40(12):2244 - 2252, 2008.

[3] A. Dröscher, M. Pichler, H. Schranzhofer, A. Constantin, N. Exizidou, G. Giannakis, and D. Rovas. Deliverable D2.2: Validation Results of the Models (Draft Version). PEBBLE 248537, FP7-ICT-2009-6.3, 2010.

[4] Eurostat. Supply, conversion, consumption - all products database. In Energy Yearly Statistics 2008, 2011.

[5] H. E. Feustel, B. V. Smith, V. Dorer, A. Haas, and A. Weber. COMIS 3.1 - User's Guide. EMPA Dübendorf, 2001.

[6] R. Z. Freire, G. H. Oliveira, and N. Mendes. Predictive controllers for thermal comfort optimization and energy savings. Energy and Buildings, 40(7): 1353 - 1365, 2008.

[7] H. Gerd, K. Jan, and S. Dietrich. Technical measurement investigations of an office building characterised by low energy use and high thermal comfort. 7th Nordic Building Physics Symposium, Proceedings, page 8, 2005.

[8] G. Giannakis, G. Kontes, E. Kosmatopoulos, and D. Rovas. A modelassisted adaptive controller fine-tunning methodology for efficient energy use in buildings. In Mediterranean Conference on Control \& Automation, 2011. To appear.

[9] M. Gwerder, D. Gyalistras, F. Oldewurtel, B. Lehmann, K. Wirth, V. Stauch, and J. Tödtli. Potential assessment of rule-based control for integrated room automation. In 10th REHVA World Congress, Sustainable Energy Use in Buildings - Clima 2010, Antalya, 2010.

[10] E. Kosmatopoulos. CLF-based Control Design for Unknown MultiInput Nonlinear Systems with Good Transient Performance. IEEE Transactions on Automatic Control, 55(11), Nov. 2010.

[11] M. Krause and S. Rodriguez. Draft Evaluation Plan. PEBBLE Deliverable 7.1, 2010.

[12] J. Liang and R. Du. Design of intelligent comfort control system with human learning and minimum power control strategies. Energy Conversion and Management, 49(4):517 - 528, 2008.

[13] A. Mahdavi, C. Pröglhof, M. Schuss, and K. Orehounig. Passive Cooling in existing buildings: an innovative approach. REHVA Journal, 47(4):42-47, 2010.

[14] F. Oldewurtel, D. Gyalistras, M. Gwerder, C. N. Jones, A. Parisio, V. Stauch, B. Lehmann, and M. Morari. Increasing energy efficiency in building climate control using weather forecasts and model predictive control. In 10th REHVA World Congress, "Sustainable Energy Use in Buildings", Clima 2010, Antalya.

[15] F. Oldewurtel, A. Parisio, C. Jones, M. Morari, D. Gyalistras, M. Gwerder, V. Stauch, B. Lehmann, and K. Wirth. Energy efficient building climate control using stochastic model predictive control and weather predictions. In American Control Conference (ACC), 2010, pages 5100 - 5105. IEEE, 2010.

[16] K. Schlegel. Documentation and analysis of an innovative research and demonstration building. Master's thesis, University Kassel, 2001.

[17] V. Vapnik, S. Golowich, and A. Smola. Support vector method for function approximation, regression estimation, and signal processing. In Advances in Neural Information Processing Systems, 1996. 